Original Research Paper

\title{
Design a New Bidirectional Transmission Protocol to Improve the Performance of MAC Layer Based on Very High Speed WLANs
}

\author{
${ }^{1}$ Ali Ahmad Milad, ${ }^{1}$ Zul Azri Bin Muhamad Noh, ${ }^{1}$ Abdul Samad Shibghatullah, \\ ${ }^{1}$ Mustafa Almahdi Algaet and ${ }^{2}$ Aouache Mustapha \\ ${ }^{I}$ Department of System and Computer Communication, Faculty of Information and Communication Technology, \\ Universiti Teknikal Malaysia Melaka, Hanngtuha Jaya, 76100 Durian Tunggal, Melaka, Malaysia \\ ${ }^{2}$ Department of Electrical, Electronic and System Engineering, Faculty of Engineering and Built Environment, \\ Universiti Kebangsaan Malaysia, Bangi 43600, Selangor, Malaysia
}

\author{
Article history \\ Received: 17-08-2014 \\ Revised: 20-01-2015 \\ Accepted: 15-06-2015 \\ Corresponding Author: \\ Ali Ahmad Milad \\ Department of System and \\ Computer Communication, \\ Faculty of Information and \\ Communication Technology, \\ Universiti Teknikal Malaysia \\ Melaka, Hanngtuha Jaya, \\ 76100 Durian Tunggal, \\ Melaka, Malaysia \\ Email: ali106014@yahoo.com
}

\begin{abstract}
This paper presents a new bidirectional transmission protocol with single data frame to computing the performance of MAC layer based on IEEE $802.11 \mathrm{n}$. As high as $600 \mathrm{Mbps}$ of physical data rate is achieved in IEEE 802.11n where high data rate the current MAC layer leads to high performance overhead and low performance of throughput and designing the MAC layer still ongoing to achieve high performance throughput. In this study, a new bidirectional transmission protocol with single data frame has been proposed called BTDF bidirectional transmission data fragmentation, which is divided each data frame from sender to receiver and reverse into subframes and send each subframe, Packets those exceed the size threshold are divided into fragments also where the corrupted subframe will be retransmitted during the disruption of transmission. We have implemented this scheme in NS2 simulator to show the results for TCP and HDTV traffics and compared with literature.
\end{abstract}

Keywords: MAC Layer, IEEE 802.11n, Reverse Direction Transmission, Subframe

\section{Introduction}

IEEE 802.11-based LAN increases everywhere to support many applications using TCP, UDP and VOIP. There is a chance of wireless LANs with high speeds where as high as $600 \mathrm{Mbps}$ could be reached for fast rate at MAC layer. The increase in the physical rate can result into increasing the transmission at MAC link, thus, causing an increase in the overhead Fig. 1. In IEEE802.11, the throughput does not scale well with increasing the physical rate (Xiao and Rosdahl, 2003; Milad et al., 2013a; Saif et al., 2011; Li et al., 2009; Abichar and Chang, 2013). However, in IEEE802.11n, the throughput achieves $100 \mathrm{Mbps}$ at a MAC layer.

IEEE 802.11 proposes a set of procedures for WLANs. One of the standards established for physical layer (PHY) specification and Medium Access Control layer (MAC) was 802.11 (Nandhini and Devarajan, 2013). Changes and development are continuously being issued for this standard by the working group. Letters "a" through "n" was issued for Task Groups (TGs) under the Working Group (WG) of 802.11. However, the advanced MAC solutions could not be utilized for the high-volume physical layers where minimization of overhead could not be succeeded which results in the limitation of MAC throughput. Hence, to aid the throughputs of 100 Mbps MAC via PHY-layer and MAC-layer supports, the IEEE 802.11n TG was issued for the development of a standard, IEEE 802.11n to replace the existing one (Bruno et al., 2008; Ozdemir et al., 2006; Zhai et al., 2004).

For achieving a wide performance enhancement or improvement, it is important to find out the major problem which causes MAC inefficiency as displayed in Fig. 2. The theoretic throughput higher limit and the theoretic delay lower limit already presents in the IEEE 802.11 protocols. Those limits show that it is possible to increase the data rate without having to reduce the overhead (Xiao and Rosdahl, 2002; Li et al., 2009; 2006).

This study aims to come up with a methodical comprehension of the three main improvised processes of IEEE 802.11n MAC layer which include aggregating 
scheme, block acknowledgment and reversed direction transmission. In past researchers, focus was given on the improved throughput by assessing the standard's efficiency and the aggregation process (Lin and Wong, 2006; Skordoulis et al., 2008; Xiao, 2005). For the outcome of this study, a comprehensive discussion and evaluation will be provided on the reverse direction transmission using single data frame in the $802.11 \mathrm{n}$ and the implementation of a new scheme which is divided the data frame from sender and receiver into subframes and send each sub- frame. Packets with capacity exceeding the limit are fragmentized also, where the corrupted subframe will be retransferred during disruption. As illustrated in Fig. 3.

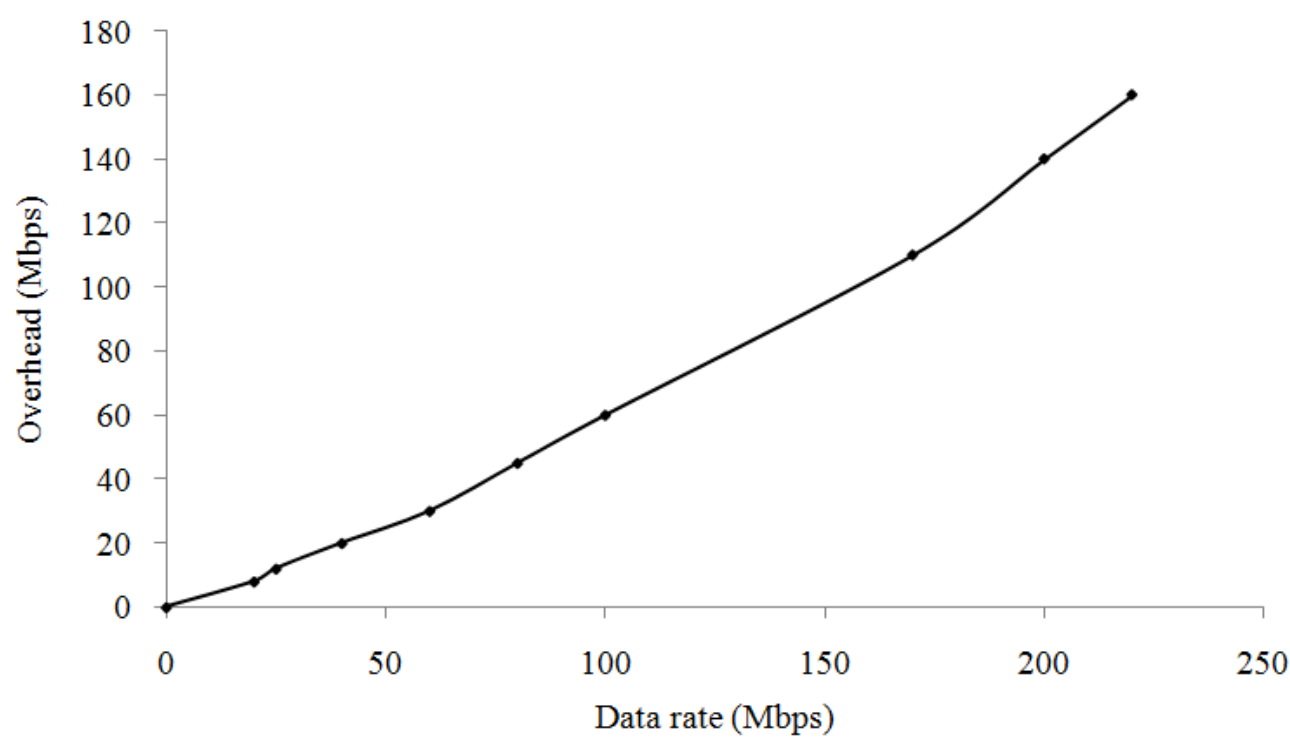

Fig. 1. Increase overhead with increase data rate

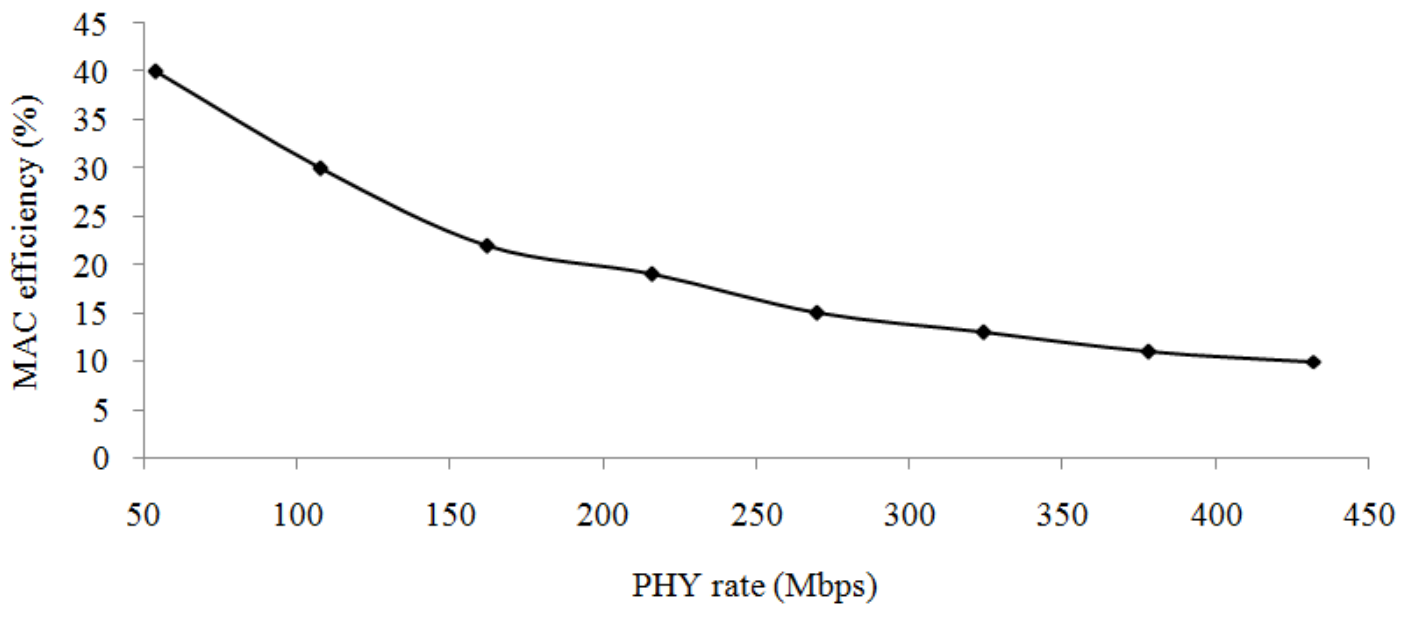

Fig. 2. MAC inefficiency in the ideal case

In our scheme, we also companied the aggregation scheme, block ACK, reverse direction transmission using single data frame and added the fragmentation method to produce a new protocol namely Bidirectional Transmission Data Fragmentation (BTDF) to Improve the performance of MAC layer.

The 802.11 Task Group (TGn) has put forward a discussion on evaluating the performance of enhanced
MAC layer of IEEE 802.11n in the perspective network effectiveness. This is a basic issue for the design of MAC in terms of cross-layer connection where the throughput from the existing 802.11 MAC is not in a good fit with the escalating rates of PHY. Given this, the characteristics of MAC scaling are given the main focus on PHY improvement and the requirement for high rates of throughput. 


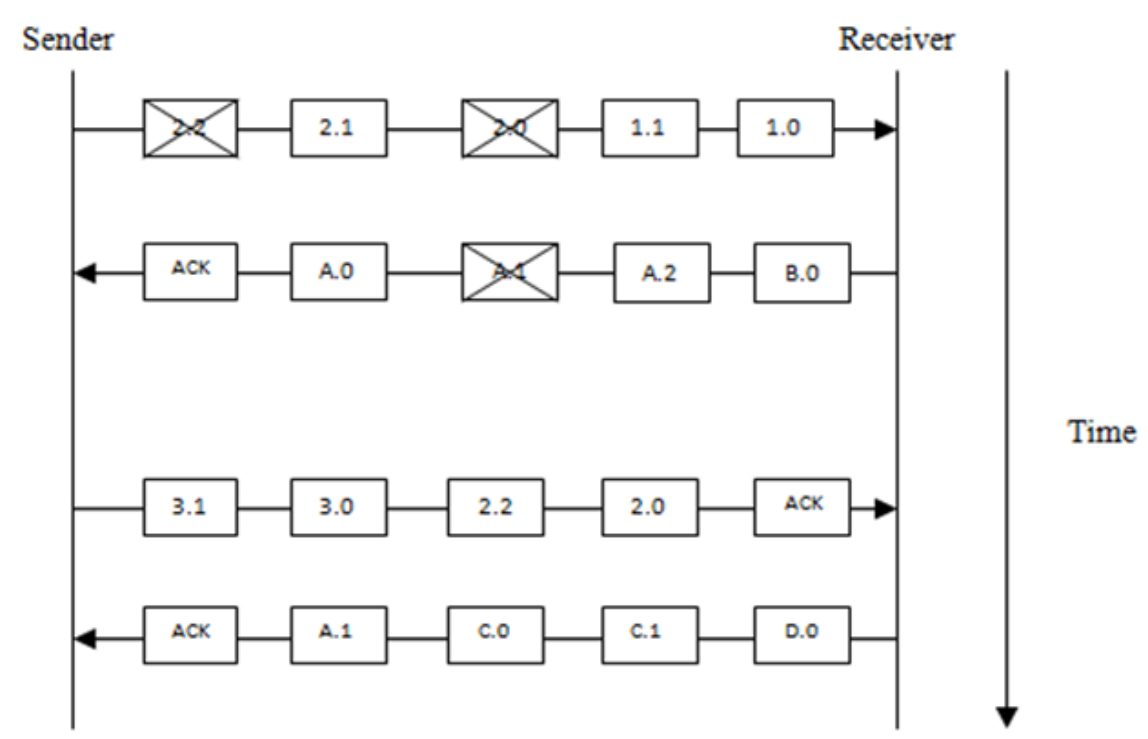

Fig. 3. BTDF protocol with single data frame

\section{Data/ACK Frame Exchange}

Data transmittance through a wireless medium is free from disruption due to a latent period and connection of repetitive process that stimulates frame re-transmittance which fails to be demodulated at the recipient. To obtain this fundamental process, a receiving station for receiving data frame and sending a simultaneous and positive ACK frame recognition should be erected. Retransmission of data may occur when ACK frame is absent at by the station that sends the data (Perahia and Stacey, 2008; Perahia, 2008).

Figure 4 shows the transferring of data via Data/ACK exchange. The illustration shows that data is being transferred from STA 1 to STA 2. Following a contention, the medium is accessed by STA 1and adjourns for DIFS and follows by random backoff duration. Data is retransmitted to STA 2 if the medium continues to be in an inert state. Following a detection and demodulation of the frame by STA 2, a reaction is followed with ACK. After receiving ACK, STA 1 acknowledges the frame and starts accessing the channel again for the next transmittance. In the condition where frame demodulation is not succeeded by SAT 2, ACK will not be received by STA 1 and retransmittance will be attempted again for the next transmittance (Hoefel, 2009).

On a normal MAC Service Data Unit (MSDU) which is received from the logical Link Control (LLC), the trials of re-transmittance are restricted. The number of attempts made is recorded by the transmittance station on MSDU and discarded it when the number of attempts exceeded the determined limit. To increase the reliance of frame recognition, the ACK frame is actively regulated such as applying a low rate of $\mathrm{PHY}$ as compared to frames transmitted to the identical station. The overhead added induced with active regulation is rather small to match the small ACK frame used (Perahia and Stacey, 2008).

\section{Fragmentation Method}

To divide a big MSDU, the fragmentation method is employed for improving the probability of receiving MSDU and in reducing the overhead of re-transmittance. Undivided MSDU uses up a large capacity of air time during low data rate. For instance, $12 \mathrm{~ms}$ is required for the transmittance of 1500 byte data frame via $1 \mathrm{Mbps}$ 802.11 b rate which results in high probability of alteration of channel conditions. The whole frame will be retransferred prior to a small error in the frame. Fragmentation enables MSDU to be divided and enclosed in MSDU and transferred in individual PPDU which is physical layer convergence procedure protocol data unit where new channel estimation is provided. MAC Protocol Data Unit (MPDU) containing the error segment will be retransferred following a small error therefore parts that make up MSDU will be transferred as separate MSDUs. Every segment may be transmitted in individual channel entry by station or as a flow of data via one channel access as shown in Fig. 5 (Chang et al., 2006). Fragmentation of MSDU is incurred when it exceeds the length as denominated by dot11FragmentationThreshold attribute. It is fragmented in an equal size beside the final segment which might be smaller and transferred according the sequence (Ho and Shaver, 2006). In the Aggregation with Fragment Retransmission (AFR) plan, various packets are combined and transferred in a large frame. In this scheme, parts with error are re-transmitted if disruption occurs during transfer (Li et al., 2009). 


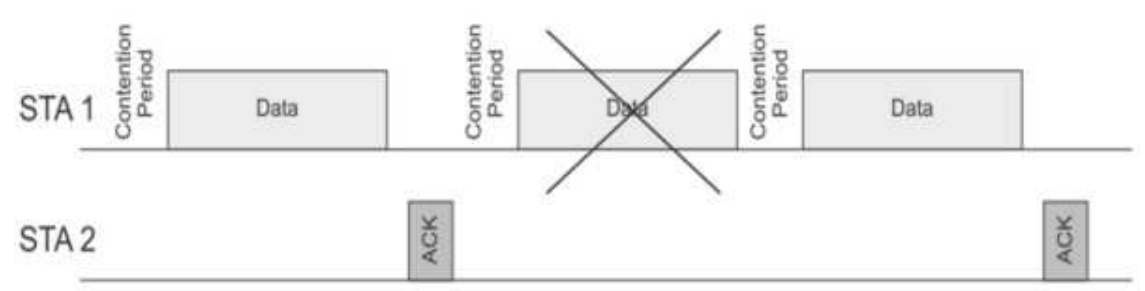

Fig. 4. Basic data/ACK frame exchange sequence

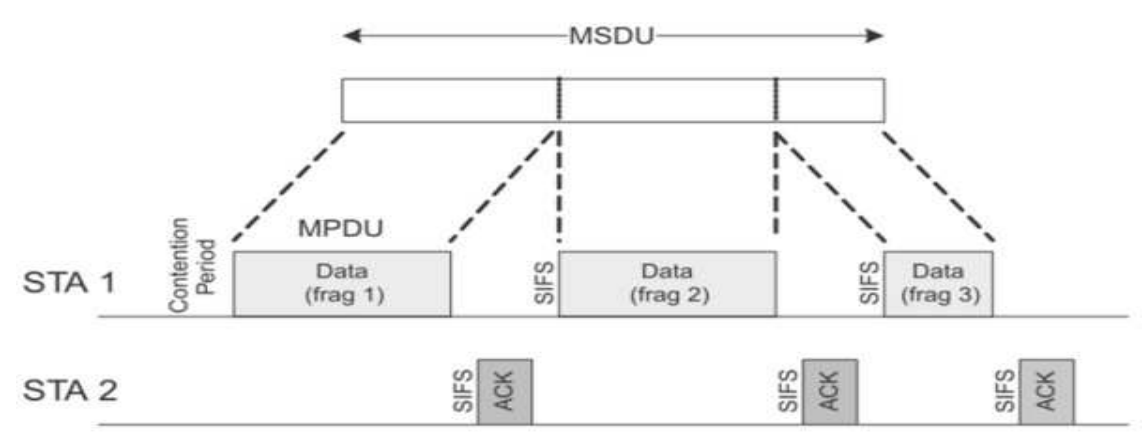

Fig. 5. Fragment burst

The transmittance of MSDU fragments occurs in a certain time limit, which is specified by the characteristics of dot11MaxTransmitMSDULifetime. The duration used for transmittance is activated during the first try. If the time limit is exceeded, the remain fragments will be eliminated (Committee WGot, 2009).

The time limit also applies for receiving MSDU fragments, which is specified by the characteristics of aMaxReceiveLifetime. The duration used for receiving the segments is activated during the first try. If the time limit is exceeded, the remain fragments that are not received will also be eliminated (Mangold et al., 2003).

\section{Major Features of 802.11n}

By using spatial multiplexing via operations of MIMO and $40 \mathrm{MHz}$, the data rates of PHY in $802.11 \mathrm{n}$ are much more efficient than 802.11a and 802.11g. To optimize this advantage, the methods of frame aggregation and development in blocking the acknowledgment procedures are used to increase the performance of MAC. The combination of these characteristics contributes towards the development of throughput via 802.11a and 802.11g.

Resilience is also enhanced via the increase in spatial diversity using multiple antennas. As with high-speed link adaptation (a process for a rapid recognition of channel alteration), the Space-Time Block Coding (STBC) is employed to increase the resilience of PHY. Greater amount of active channel codes are issued as Low Density Parity Check (LDPC) codes. Transmit beam forming is also introduced by the standardization along with PHY and MAC improvements.
Other benefits are provided by several other improvements. A shorter intervening guard is included in PHY to be utilized in specific channel conditions. A Green field preamble shorter than the specified format is also introduced. However, it does not match the current $802.11 \mathrm{a}$ and $802.11 \mathrm{~g}$ tools in the absence of MAC aid. The reversed procedure improves the efficiency for particular traffic conditions in the MAC through the reduction of total overhead channel access. It is done by permitting the station to sublet the remaining segments of its distributed transmittance to distant equals. Using Reduced Inter-Frame Space (RIFS) during the transmittance of influx of frames results in a lower overhead than the current Short InterFrame Space (SIFS).

\section{Enhancements of IEEE 802.11n}

The IEEE 802.11Working Group adopts the IEEE $802.11 \mathrm{n}$, which is a modified version for elevated throughput and rate. The main goal of IEEE 802.11n is to provide a higher throughput rather than faster rates along with PHY and improvements of Medium Access Control (MAC) (Soni and Mendiratta, 2008; Xiao, 2005). Moreover, IEEE $802.11 \mathrm{n}$ provides many enhancements to reduce the overhead at MAC layer, such as: frame aggregation, Block Acknowledgment (BA) and reverse direction transmission (piggybacking ACK) (Saif et al., 2012) as shown in Fig. 6. The Improvements of next generation wireless LANs IEEE $802.11 \mathrm{n}$ will be discussed in details especially in more concern with the reverse direction transmission. 


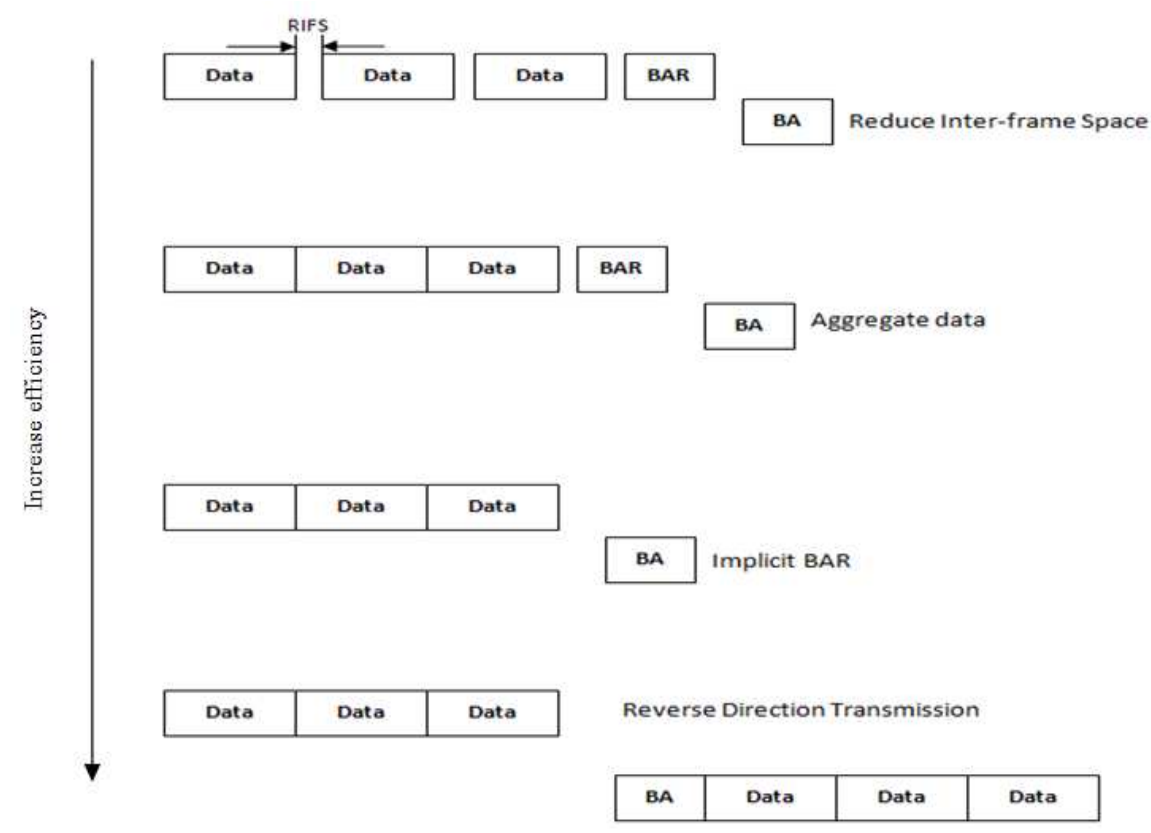

Fig. 6. Basic throughput enhancements to the IEEE 802.11n

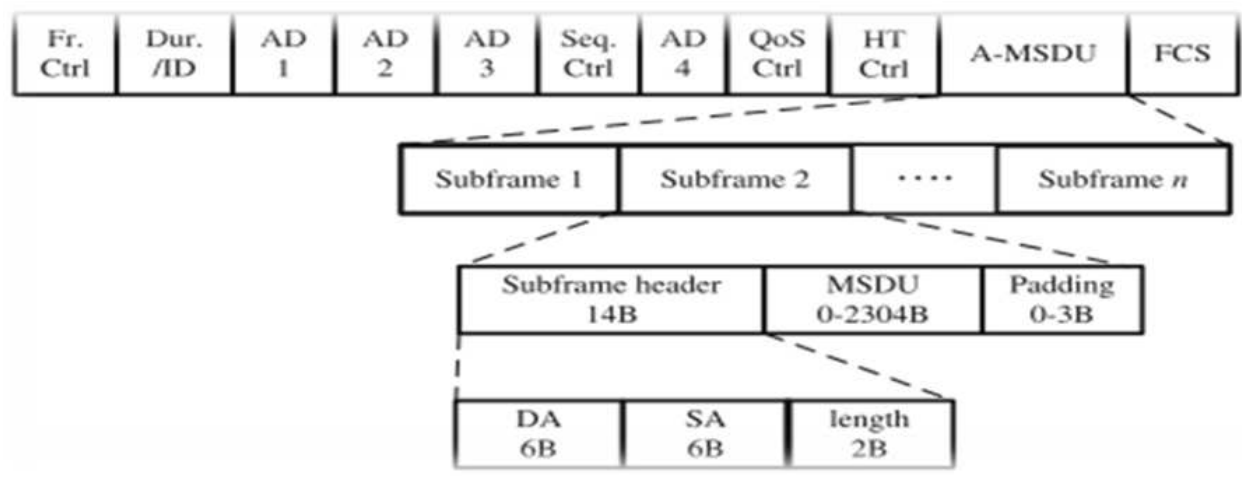

Fig. 7. Shows the frame of A-MSDU

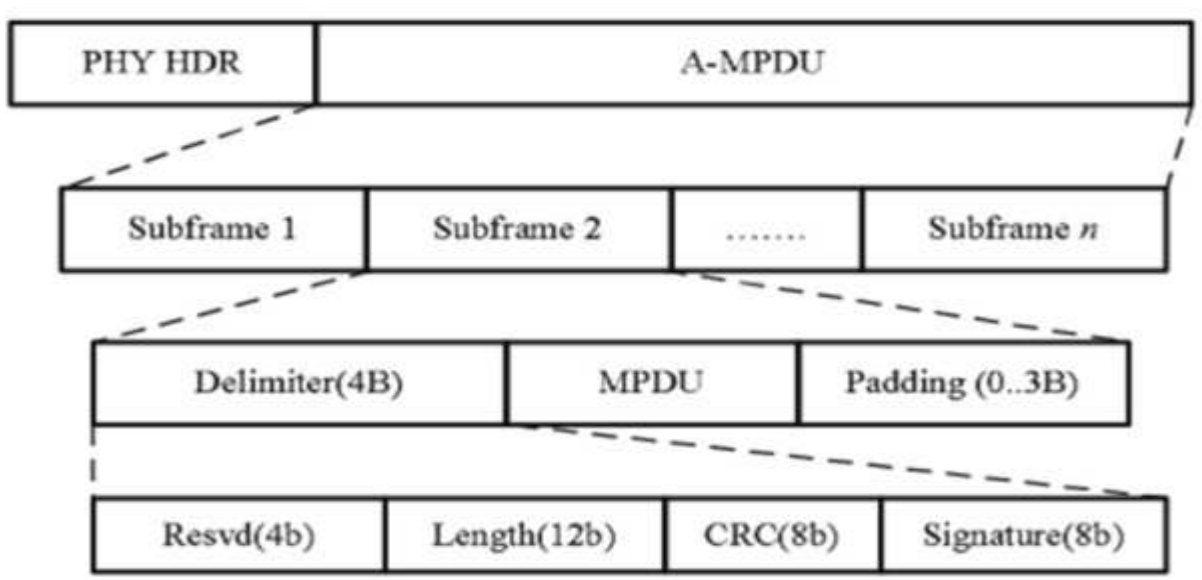

Fig. 8. Shows the frame of A-MPDU 


\section{Frame Aggregation}

Frame aggregation is defined as an application which allows combining a few outlines into a larger, individual outline for transfer. The process is carried out by using two available procedures: An aggregation of MAC custom functional block (A-MSDU) and the aggregation of MAC custom information block (A-MPDU). Moreover, an MSDU can be mainly distinguished from MPDU in terms of its information transfer functions. MSDU corresponds to the import or export of data from the topmost layer of MAC, while the latter depends on data transfer by the lower part of the MAC from or to the PHY.

The alignments of aggregation conversion are facilitated by a custom acknowledging various MPDUs containing an individual unit ACK. This is made as a feedback for the Block Acknowledgment Request (BAR). An alternative method to achieve such improvement includes the specification of $802.11 \mathrm{n}$ in the two directions of data conveyance over an individual TXOP known as opposite route. This characteristic allows the transfer of informational outlines including the aggregations of two directions in an individual TXOP. Currently, allocating a TXOP to the transmitter STA may inform adjoining STAs regarding the duration of the wireless instrument engagement. The results this estimation of medium provides, however, cannot be continuously precise; with the transfer failure is often sooner. In other cases, alleged STAs, therefore, with an expectation that the route is engaged. Moreover, with reverse direction, any available packets directed at the transmitter for the remnants of TXOP duration are allowed to be sent by the initial receiver STA. This, in particular, suits TCP well as it permits a connection of TCP to TCP ACK towards TCP delivery. Long-NAV (long network allocation vector) refers to a different progress which develops scheduling as long as the infrastructure includes a TXOP is able to erect a longer NAV for the intention of saving multiple PPDUs. A different compulsory characteristic include Phased Coexistence Operation (PCO). The main purpose of this operation is to protect stations either through $20 \mathrm{MHz}$ or 40 MHz channel range simultaneously. Lastly, a lower IFS (RIFS) for permitting a time lapse of $2 \mu$ s between various PPDUs is suggested which is identified in the legal customs as much shorter than SIFS (Skordoulis et al., 2008).

\section{A-MSDU Aggregation}

A-MSDU (MSDU aggregation) permits the sending various MSDUs towards an identical recipient in an individual MPDU. Absolutely, the process leads to enhancing the effectiveness of MAC outline, mainly during the inclusion of various small-scale MSDUs include TCP recognitions Within the $802.11 \mathrm{n}$, the enhancement of AMSDU proved to be compulsory at the receiver's side (Rasheed et al., 2012; Kolap et al., 2012).
As illustrated by Fig. 7, in the formation of an AMSDU, MAC topmost layer acquires and reserves multiple packets (MSDUs). It is fulfilled in one of these two cases: With a maximum A-MSDU threshold waiting packet capacity or when the crossing of the estimated value of postponement by oldest packet. Moreover, its maximal range is permitted to be 3839 or 7935 bytes which is 256 bytes less than the maximal range of PHY PSDU (4095 or 8191 bytes simultaneously), as the expected capacity is preserved for coming events or for controlling data. The capacity is noticed during High Throughput (HT) induced from HT STA to affirm its HT condition. Although the maximum postponement can be adjusted to a separate range for each AC, it is commonly adjusted to $1 \mu \mathrm{s}$ for every ACs. When constructing an AMSDU, certain limitations can be detected:

- The same TID value must be acquired by all MSDUs

- Capacity of A-MSDU needs to be appropriate according to the maximal capacity of its integral elements

- Identical Receiver Address (RA) and Transmitter Address (TA) must with Destination Address (DA) and Sender Address (SA) (Skordoulis et al., 2008; Saif and Othman, 2013)

Broadcasting or multicasting, therefore, is prevented. However, the main weakness of employing A-MSDU is represented by the under networks immune to errors. Furthermore, the whole A-MSDU must be retransferred, by combining every MSDU via a pattern. This case is dealt with in (Lin and Wong, 2006), where further configurations have been suggested to achieve accuracy in busy networks.

\section{A-MPDU Aggregation}

A-MPDU connects multiple MPDU sub-frames to a main PHY header, refer to Fig. 8. The only aspect in which it differs from A-MSDU is the function of AMPDU since it commences after the MAC header enclosure procedure. Because of this, the A-MSDU control of assembling frames according to appropriate TIDs is not considered a function of A-MPDUs. Nevertheless, it is necessary to deal with all the MPDUs bounded by A-MPDU using the identical recipient. Besides, the luck of waiting/holding time needed in forming an A-MPDU makes the required amount of MPDUs entirely dependent on the amount of existing packets within the transmittance sequence. Furthermore, 65,535 bytes is the highest capacity obtained by AMPDU (the highest capacity of the PSDU probably to be accepted). However, it is possible to be reduced depending on the existing STA abilities in the elemental ability of HT. Moreover, the maximum value of sub 
frames acquired amounts to 64 and this is due to the reason that capacity of unit ACK bitmap (128 bytes) where 2 bytes were used to map each frame and are needed for acknowledging up to 16 parts. However, since the A-MPDUs prevent fragmentation, these additional bits become excessive. Therefore, a new type was conducted which is called compressed block ACK with 8 bytes of bitmap field. Lastly, the capacity of every subframe confines to 4095 bytes due to the inability of PPDU length in surpassing the pre-determined time threshold at $5.46 \mathrm{~ms}$. This can obtained by dividing the maximum length with the lowest PHY value (Skordoulis et al., 2008; Hajlaoui et al., 2012).

\section{Block Acknowledgment}

A Block ACK method was recommended in the IEEE802.11e particularly in Block Transmission and Acknowledgment (BTA) scheme and therefore a block of data frames send it to the destination in sequence and between each one and the data frame there is a SIFS period of time. After sending the block of data, the sender starts sending the Block Acknowledgment Request (BAR) to ask about frame which has been received by the receiver.

Then, the Block Acknowledgment (BA) is redirected to the transmitter including information about the obtained frame as illustrated in Fig. 9. When the block acknowledgement is received correctly, the sender is supposed to delay the DIFS and back off mechanisms earlier than sensing the channel and all other stations should wait until the block acknowledgment transmission finishes. If many stations have started transmitting the data with BAR at the same time, the collections will occur. Moreover, when each station sends the data with BAR, the receiver should wait to detect the collision first and then send the block acknowledgment (Saif et al., 2011; Ghaleb et al., 2012).

\section{Reverse Direction Transmission}

Two different categories of infrastructures are identified in reverse direction operation: RD initiator and $\mathrm{RD}$ responder. The former is the infrastructure that supports the TXOP and permits sending Reverse Direction Grant (RDG) received by the responder. Furthermore, RDG is labeled in the header of $802.11 \mathrm{n}$ and transmitted to $\mathrm{RD}$ responder along with the information frame. Upon receiving the information frame along with $\mathrm{RDG}$, the $\mathrm{RD}$ responder reacts by acknowledging RDG mainly if there is information that needs to be transmitted or when RDG is absent when no data is available to be transmitted. Then, the RD initiator needs to hold back for the RD responder transfer when the acknowledgement is marked with RDG (Wang and Wei, 2009; Lim and Suh, 2010; Abichar and Chang, 2013) Fig. 10.

\section{Reverse Direction Transmission Approaches}

The $802.11 \mathrm{n}$ RD protocol mainly aims to accurately exchange the information among two 802.11 tools throughout a TXOP by restricting the necessity of each tool to have access for further information exchange. Prior to RED custom, each transmittance of single directional information needs the starting infrastructure to attract (include the possibility of time reservation) a allegation-based RF medium. Other infrastructures and stations are essentially allowed to send information back with RD once the transmitting station has attained a TXOP. Therefore, two functions need identification namely RD initiator and RD responder. According to a Reverse Direction Grant (RDG) in the RDG, RD initiator transmits its acknowledgment to the RD responder. More PLCP Protocol Data Unit (PPDU) field of the HT Control is as implied in Fig. 11. The RD responder adopts this bit to grant access (RDG) and is used by the RD responder to prove if it can send more immediate frames subsequently after it has just obtained (more PPDU) (Milad et al., 2013a).

\section{Reverse Direction (RD) Exchange Sequence}

An RD exchange sequence includes:

- The transfer of the PPDU by the TXOP holder including an RD grant (the RDG PPDU) as indicated by the PPDU consists of one or more high throughput control (+HTC) MPDUs where RDG/More PPDU subfield has been placed into 1 . The STA transmitting this PPDU is called the RD initiator. The process of carrying out the rules for an $\mathrm{RD}$ initiator is performed only during a single RD exchange sequence (e.g., after the transfer of an RDG PPDU till the end of the last PPDU in the RD exchange sequence)

- The transfer of one or more PPDUs (the RD response burst) by the STA is dealt with in the MPDUs of the RDG PPDU. The first (or only) PPDU of the RD response burst includes at most one immediate Block ACK or ACK response frame this process is called piggybacking. The last (or only) PPDU of the RD response burst includes any MPDUs requiring an immediate Block ACK or ACK response. The RD responder is the STA that transfers the RD response burst. Furthermore, It is only during a single RD exchange sequence that the rules for an RD responder can be applied, i.e., following the reception of an RDG PPDU and up to the transfer of the PPDU by the RD responder in which the RDG/More PPDU subfield is put into 0

- The transfer of the PPDU by the RD initiator containing an immediate Block ACK or ACK MPDU (the RD initiator final PPDU), If so needed by the last PPDU of the RD response burst 


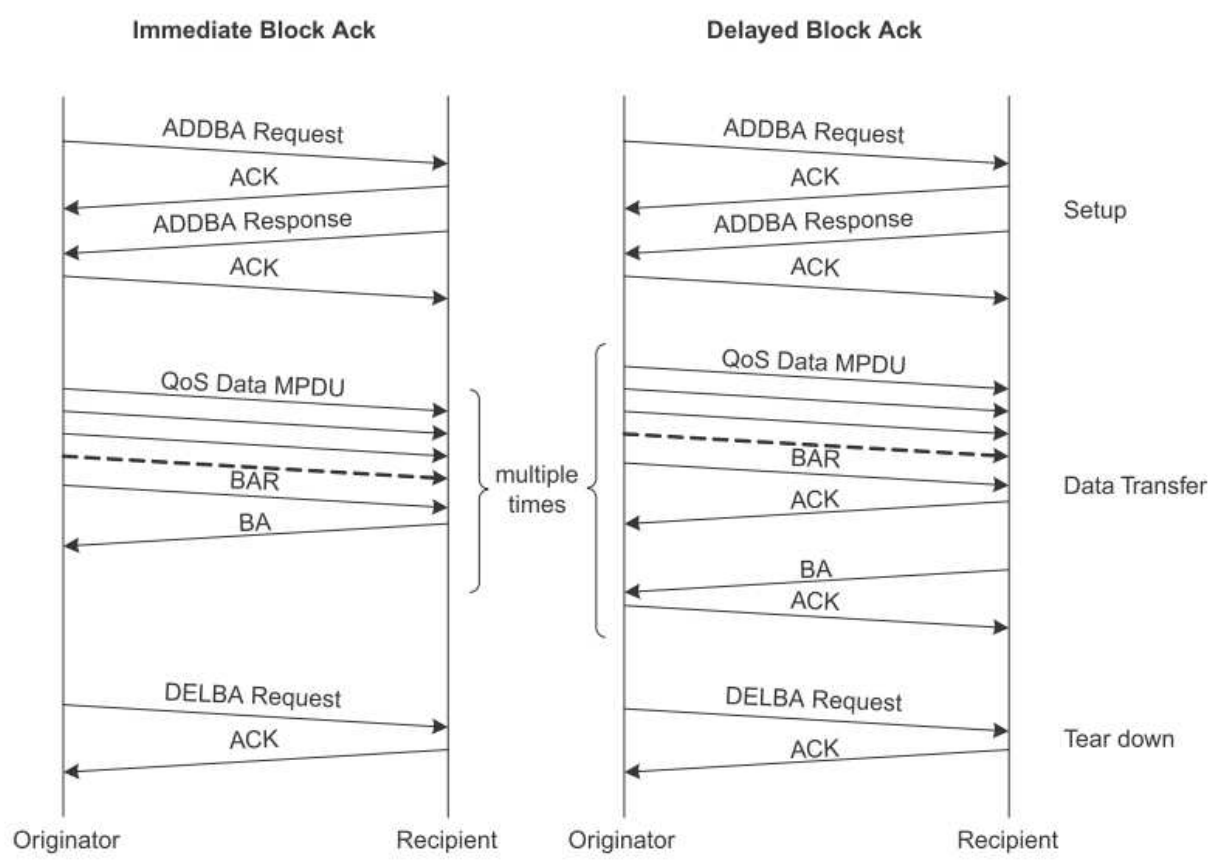

Fig. 9. Immediate and delayed block acknowledgment

Remaining TXOP (1)

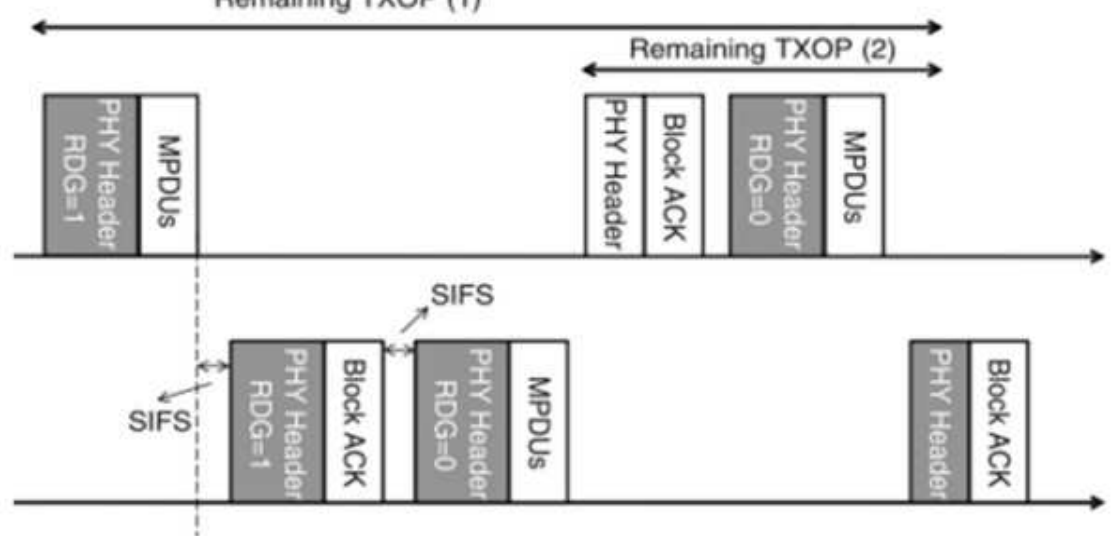

Fig. 10. Reverse direction transmission

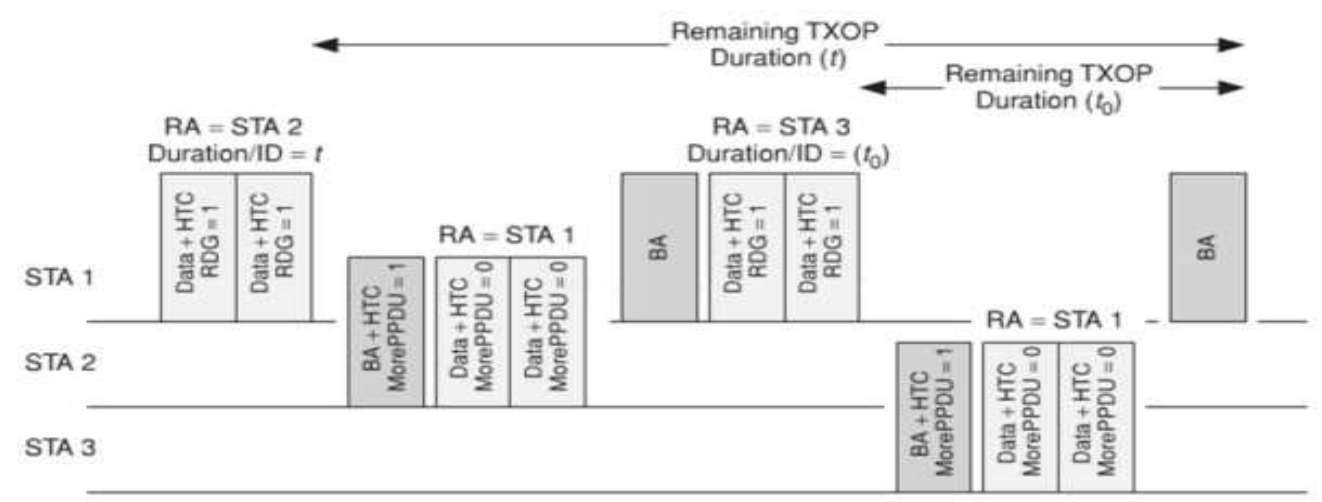

Fig. 11. Revere direction exchange 
NOTE: The RD initiator may contain multiple RD exchange sequences within a single TXOP. Each RD exchange sequence within a single TXOP that can be dealt with by different recipient and it is probable that any single recipient includes more than one RDG within a single TXOP (Milad et al., 2013a).

\section{Bidirectional Transmission with Single Data Frame}

When the data packets are transferred in duplex directions from part $\mathrm{A}$ to part $\mathrm{B}$ and the data arrives at $\mathrm{B}$, instead of sending a control frame from $B$ to $A$, receiver $B$ waits until the network layer at $B$ sends the next packet to $A$ and the acknowledgment is attached in the data frame from $\mathrm{B}$ to $\mathrm{A}$ using the field of acknowledgment in the data frame header. Therefore, the acknowledgment gets a free ride in the data frame and this technique is known as piggybacking. One of the most well-known advantages of piggyback scheme is improving the efficiency, which plays the role in reducing the overhead and increasing the system throughput (Milad et al., 2013b; 2013c).

When the recipient infrastructure has a frame to the transmittance infrastructure and allows sending the data frame with ACK to the sender, this process is called a piggyback scheme. So the acknowledgment gets a free ride in the data frame and takes few bits and this is a distinct ACK. Therefore, each frame requires an ACK header and data frame (checksum). This means that the piggyback merely relies on the receiver since the fewer the frames are sent, the fewer the frames that arrive and this is dependent the way the receiver is organized. However, the emergence of the piggyback scheme has posed some cases of complexity. For example, the question of how long the data link layer is supposed to wait until the packet is transmitted to piggyback the ACK is still a posing a complex case. As far as we know, the link layer usually waits for a certain period of time and if this waiting period consumed by the data link layer is longer than the sender timeout period, it is expected that the frame will be retransmitted. Therefore, it is assumed that the data link layer must wait for fixed time like $\mathrm{Ad}$ hoc scheme such as number of milliseconds. On the other hand, one of the benefits of the piggyback is that the piggyback frame does not need to rivalry the channel again in a case when the receiver has a frame to transmit to the transmitter. It is because it does not need to be in the front of the queue but the nearest frame to the destination at the sender. In (34) the researcher shows the overhead with/without piggyback mechanism in case when the frame belong to the receiver is sent to the sender after receiving a frame as completion of the channel. After this a RTS and CTS frame time, DIFS time, two SIFS and a random back off is required to be done by it. Otherwise, in a case when the frame is possible to be piggybacked by the receiver to the sender along with the acknowledgment, the ACK is sent by the sender as a way of acknowledging the piggybacked frame after reducing the SIFS time and the overhead has been already accomplished Fig. 12 .

In IEEE 802.11 WLAN data frame improves wireless network performances. But the piggyback will reduce network performance for frame transmittance postponement. In Global control information the stations have a low rate of transmittance and in control frames. So the piggyback deals with the problem concerning the low rate of transmittance, evaluation of the impact induced by an average frame transmission delay and the network usage. Therefore, the purpose of proposing the delay-based piggyback scheme by the authors was to mitigate the piggyback problem (Lee et al., 2007a). As revealed by the same authors, the piggyback led to decreasing the channel efficiency while increasing the transmission delay for infrastructure with low rate of transmittance were present.

Lee et al. (2010) investigated the piggyback scheme when the data frame was transmitted in two cases for the station. If the frame was corrupted, so whole the process would re-start, or the data frame would be received successfully. The researcher also showed that the network infrastructure switches to piggyback scheme when the packet uses below 1100 bytes. In addition, the throughput is very low because the overhead is high and the data rate is better than the piggyback as duration missed in "data+Acknowlegment" frames retransmittance is deducted.
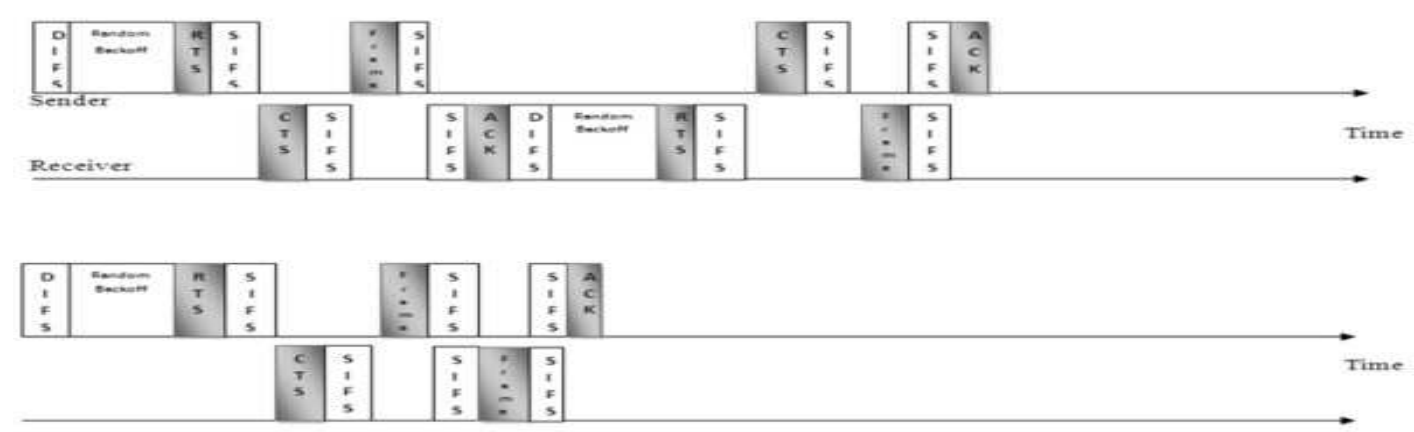

Fig. 12. The overhead with/without the piggyback scheme 
Lee et al. (2007b) defined the piggyback problem as when the main frame is piggybacked, the channel efficiency is decreased and at the same time, the infrastructure emits a minimal rate. The researchers also showed that and evaluated the network application by including the piggyback frame and they proved that utilization of channel results in the ratio for time required for transmittance to the super frame. So they solved the piggyback issue as the poor physical rate by calculating the scheme's delay and discovered that piggyback was decreasing the network performance while increasing the postponement of frame transmittance even in one station physical rate. However, when the physical transmission rate increases, it results in the increase of channel efficiency. Therefore, if the piggyback practices well, the network performance and the delay transmission will improve.

Park et al. (2009) Showed that the processes of piggyback decreases uplink and downlink packet delays. It also showed the reduction of the possibility in loss in uplink and downlink traffic using piggyback and backoff schemes. Packets do not occur for piggyback method for downlink data while in backoff, the loss packets are considerably large. Moreover, the piggyback method induces significant energy usage and no loss of downlink data packets has been shown.

Milad (2014) Examine the performance enhancement of the proposed $802.11 \mathrm{n}$ MAC layer in terms of reverse direction transmission using a single data frame and multi data frames, the analysis evaluated the throughput and efficiency delay of bidirectional transmission with single data frame and reverse direction transmission with multi data frames in noisy channels and compared to the identical procedures in the previous studies.

\section{Simulation Module Implementation}

It is obvious that the development of feasible BTDF format focuses on the latest development and new configurations of ACK frame. Problems encountered for the developments of latest configuration are acknowledging the obstacles on overhead as stated and making sure the retrieval of precise fragment transmitted during errors, which is hard as the receiver may not know the size of failed fragments. A frame header and body are consisted in MAC frame in our scheme as shown in Fig. 13.
In distributed coordination function DCF MAC; all fields in header are retained with three additions (fragment number, its size and an extra field) in new MAC header. In MAC frames, fragments used are represented by fragment sizes and the amount of fragments are represented by the amount of existing fragments. The extra field is reserved for expansion and the maintenance of alignment in the future. The headers, bodies and its respective Frame Check Sequences (FCS) are consisted in the frame body Fig. 14 and 15.

The header consists of different sizes (between 1 and 256 fragments) where every fragment is guarded by FCS. Its length is fixed at $8 \mathrm{~B}$ and is acknowledged by both transmitter and receiver.

Every fragment in the frame can be located even in the case of corruption as the beginning and size of fragment header is known by receiver.

Six fields are consisted in every fragment (packet length ( $p L E N)$, packet ID (pID), offset, startPos, FCS and spare). The respective ID together with its size of packet $\mathrm{P}$ of the segment is represented by $\mathrm{pID}$ and pLEN. startPos identifies the location of fragment body within the frame and is recorded by offset via packet $\mathrm{P}$. ACK's latest configuration is simple with the addition of 32-B bitmap in of ACK and every bit is utilized to identify the fragment precision, Fig. 16.

To explain how this protocol is work, let's give this example: i.e.,: The maximum data frame is 2048 B and we have three packets need to send with different lengths of $1025 \mathrm{~B}, 200 \mathrm{~B}$ and $60 \mathrm{~B}$ and the subframe length is $512 \mathrm{~B}$, then the algorithm will divides these packets into 3,1 and 1 subframes respectively, then send it them. For correctness subframe received will indicator at ACK bit map and the corrupted subframe will retransmitted:

- $\quad$ Subframe 1: Packet ID =1, packet length 1025 , start pos $=0$, offset $=0$

- Subframe 2: Packet ID = 1, packet length 1025, start pos $=512$, offset $=1$

- $\quad$ Subframe 3: Packet ID = 1, packet length 1025, start pos $=1024$, offset $=2$

- $\quad$ Subframe 4: Packet ID = 2, packet length 200, start pos $=1025$, offset $=0$

- $\quad$ Subframe 5: Packet ID = 3, packet length 60, start pos $=1225$, offset $=0$

\begin{tabular}{|c|c|c|c|c|c|c|c|}
\hline 38 & $8 \cdots 2048$ & $64 \cdots 2048$ & 2 & $64-\cdots 2048$ & 2 & $64-\cdots 2048$ & 2 \\
\hline MAC header & Fragment headers & Fragment 1 & FCS & Fragment 2 & FCS & Fragment $\mathrm{N}$ & FCS \\
\hline
\end{tabular}

Fig. 13. Data frame fragmentation

\begin{tabular}{|c|c|c|c|c|c|c|c|c|c|c|}
\hline 2 & 2 & 6 & 6 & 6 & 2 & 6 & 2 & 1 & 1 & 4 \\
\hline $\begin{array}{l}\text { Frame } \\
\text { Control }\end{array}$ & Duration & Address 1 & Address 2 & Address 3 & $\begin{array}{c}\text { Sequence } \\
\text { Control }\end{array}$ & Address 4 & $\begin{array}{c}\text { Fragment } \\
\text { Size }\end{array}$ & $\begin{array}{l}\text { Fragment } \\
\text { Number }\end{array}$ & Spare & FCS \\
\hline
\end{tabular}

Fig. 14. MAC header 


\begin{tabular}{|c|c|c|c|c|c|c|c|c|c|c|c|c|}
14 bits & 14 bits & 2 & 1 & \multicolumn{1}{c}{14 bits } & 1 & \multicolumn{1}{c}{14 bits } & 14 bits & 2 & 1 & 4 bits & 1 \\
\hline $\begin{array}{c}\text { Packet } \\
\text { ID }\end{array}$ & $\begin{array}{c}\text { Packet } \\
\text { length }\end{array}$ & $\begin{array}{c}\text { Start } \\
\text { Pos }\end{array}$ & Offset & Spare & FCS & $\ldots--$ & $\begin{array}{c}\text { Packet } \\
\text { ID }\end{array}$ & $\begin{array}{c}\text { Packet } \\
\text { length }\end{array}$ & $\begin{array}{c}\text { Start } \\
\text { Pos }\end{array}$ & Offset & Spare & FCS \\
\hline
\end{tabular}

Fig. 15. Fragment headers

2

\begin{tabular}{|l|l|l|l|l|}
\hline Frame control & Duration & Receiver address & Fragment bitmap & FCS \\
\hline
\end{tabular}

Fig. 16. Acknowledgment format in BTDF protocol

\section{Maximum MAC Frame Size}

According to the standard 802.11n (Committee WGot, 2009) noted that the maximum MAC frame size is 65536 bytes and our target to achieve high system throughput when the maximum data frame is 32768 bytes and the data rate more than $216 \mathrm{Mbps}$, while maximum data frame 65536 bytes when data rate up to $648 \mathrm{Mbps}$.

\section{Optimal Sub-Frame Size}

The subframes play a central role in BTDF especially in retransmission, only spoiled or damaged bits are retransferred during the utilization of tiny fragment size, however its overhead is relatively large because every part has an accurate header. Applying a large fragment results in small overhead, however as the entire fragment may be retransferred owing to a single corrupted bit in a fragment; many bits will be unnecessarily retransferred. Since it is expected to be $\left(128,512\right.$ and 1024 bytes for $B E R=10^{-4}$, $\left.10^{-5}, 10^{-6}\right)$, the optimal fragment size relies on the BER.

\section{Simulation Results}

In network simulator NS2, the BTDF scheme is applied. The network topology utilized is a peer-to-peer one which packets were sent to STA $\mathrm{i}+1$ by STA. Results for two categories of network is reported, which include TCP and HDTV which is adhered to the standardizations of $802.11 \mathrm{n}$. The efficiency of BTDF is studied in various channel measurements include the amount of transmission stations, fragment capacity as well as BER. The parameters used in NS2 have been clarified in Table 1 .

The following metrics have been used to evaluate the performance:

- Throughput: Throughput represents the maximum rate at which the MAC layer can forward packets from senders to receivers and reverse in a given amount of time. The performance of throughput in the results graphs achieved by all the system instead of a single station
Let $C$ : Denote number of packets, $L_{p}$ : Denote the packet size, $T$ : Represent the simulation time. Throughput $=C^{*} L_{p} / T$ Mbps.

- Delay: is a time by successfully transmitted a packet in reverse direction

\section{TCP Traffic}

It is evident that the majority of the issues concerned with the performance of the TCP/IP networks are resulted from a variety of interaction patterns between the TCP engine and the surrounding communication environment (Tam and Wang, 2003; Fayyaz et al., 2013). Therefore it is very important to explore the upholding of BTDF for TCP traffic.

First we find out the BTDF throughput performance with different fragment size and compare it with AFR scheme, Normal bidirectional transmission BT and with the standard DCF as shown in Fig. 17. BTDF protocol got the highest performance compared with the literature, When $\mathrm{BER}=10^{-4}$ the throughput of BTDF achieves around $42 \mathrm{Mbps}$ and increased when $\mathrm{BER}=10^{-5}$ to $70 \mathrm{Mbps}$ and when $\mathrm{BER}=10^{-6}$ has been achieved up to 104 Mbps. The increase in BTDF compared to AFR ranged at $12 \mathrm{Mbps}$ at $\mathrm{BER}=10^{-4}$ to $2 \mathrm{Mbps}$ at $\mathrm{BER}=10^{-5}$ to $23 \mathrm{Mbps}$ at $\mathrm{BER}=10^{-6}$.

At Fig. 18, we evaluate the performance of BTDF with number of stations from 10 to 80 stations and shows that the throughput of BTDF achieved high performance compared with the previous protocols, when $\mathrm{BER}=10^{-4}$ the throughput of BTDF achieves $30 \mathrm{Mbps}$ and increased when $\mathrm{BER}=10^{-5}$ to $100 \mathrm{Mbps}$ and when the $\mathrm{BER}=10^{-6}$ the throughput has been achieved 130 Mbps. The increase in BTDF compared to AFR ranged at $20 \mathrm{Mbps}$ at $\mathrm{BER}=10^{-4}, 10^{-5}, 10^{-6}$.

In Fig. 19, we found out the delay performance of BTDF protocol and compared with the literature protocols and got the observation that BTDF achieves lower delay than the others compared with number of sub frames, increase in the size of sub frames results in increase of delays that highly benefits BTDF. The number of stations was 10 . As a result, the maximum delay got at subframe 1024 bytes when the $B E R=10^{-4}$ 
around $400 \mathrm{~ms}$. The delay of BTDF and the literature protocols have plotted in Fig. 20. The BTDF protocol scores the lowest delay. As the result the delay increased through an increase in stations which also results in contention and super frame re-transmittance period.

\section{HDTV Traffic}

IEEE 802.11n states that the developing WLANs must be equipped with the support of HDTV (21). Properties of HDTV include the rate of 19.2-24 Mbps, peak delay demand at 200-ms peak delay requirement and constant packet size of $1500 \mathrm{~B}$. Efficiency of BTDF HDTV is studied using data rate of 432 Mbps PHY. Figure 21 illustrates the efficiency of BTDF throughput with the rest of schemes compared with STA's amount (with HDTV mechanisms) varies. BTDF and AFR schemes support 10 streams for $\mathrm{BER}=10^{-5},-6$, the improvement is clear when $\mathrm{BER}=10^{-6}$ compared with AFR with $0.03 \mathrm{sec}$ as showed in Fig. 22.

Table 1. NS2 parameters

\begin{tabular}{lll}
\hline & TCP & HDTV \\
\hline Number of stations & 50, varied & varied \\
Application rate & N/A & $19-24 \mathrm{Mbps}$ \\
Data rate & $432 \mathrm{Mbps}$ & $432 \mathrm{Mbps}$ \\
Basic rate & $54 \mathrm{Mbps}$ & $54 \mathrm{Mbps}$ \\
Frame size & 8192 bytes & 9000 bytes \\
Fragment size & Varied 128,256,1024 (bytes)... & 750 bytes \\
\hline
\end{tabular}

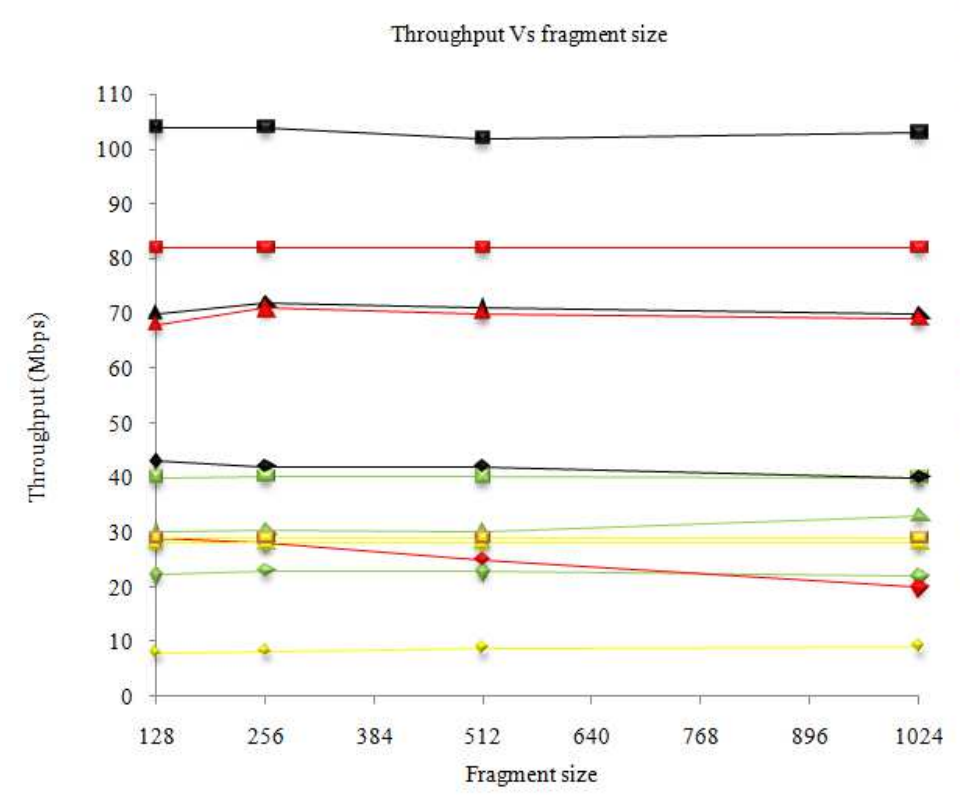

Fig. 17. Throughput compared with subframes

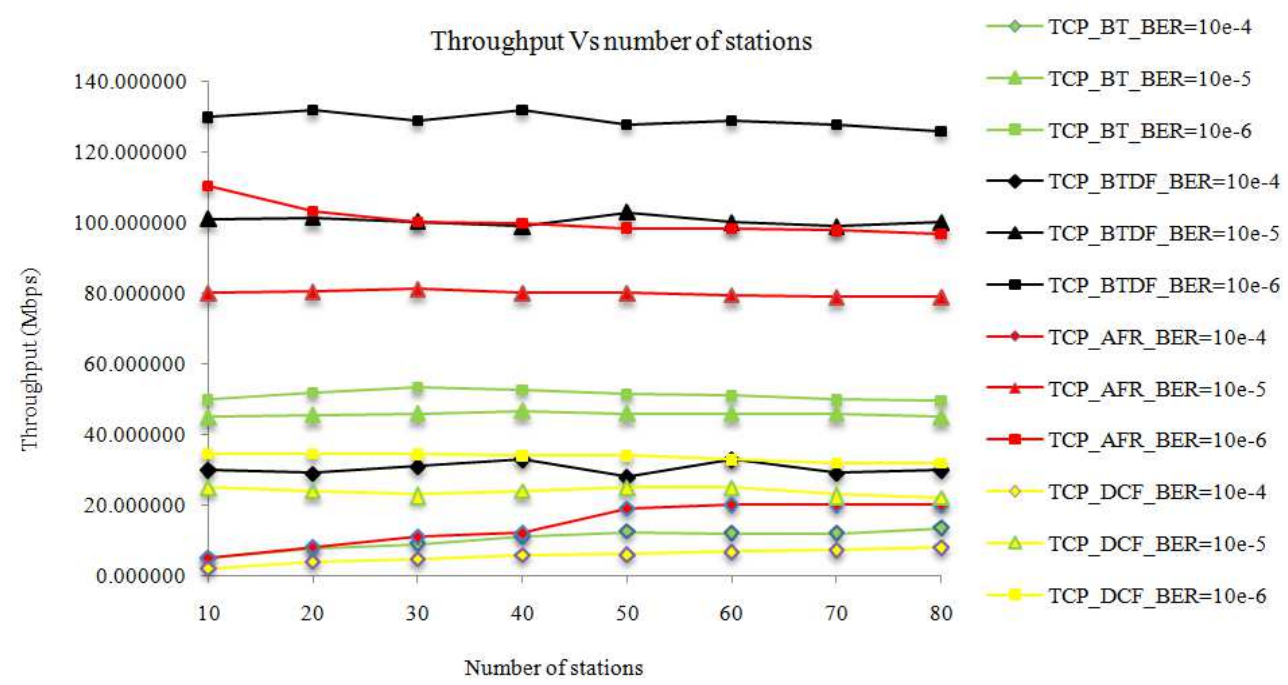

Fig. 18. Throughput compared with number of stations $\rightarrow$ TCP_BT_BER $=10 \mathrm{e}-4$

$\triangle$ TCP_BT_BER $=10 \mathrm{e}-5$

17-TCP_BT_BER $=10 \mathrm{e}-6$

$\longrightarrow$ TCP_BTDF_BER=10e-4

—TCP_BTDF_BER $=10 \mathrm{e}-5$

- $\mathrm{TCP}$ _BTDF_BER $=10 \mathrm{e}-6$

$\triangle$ TCP_AFR_BER $=10 \mathrm{e}-4$

$\triangle$ TCP_AFR_BER $=10 \mathrm{e}-5$

- TCP_AFR_BER=10e-6

$\checkmark$ TCP_DCF_BER $=10 \mathrm{e}-4$

$\triangle$ TCP_DCF_BER $=10 \mathrm{e}-5$

- TCP_DCF_BER $=10 \mathrm{e}-6$ 


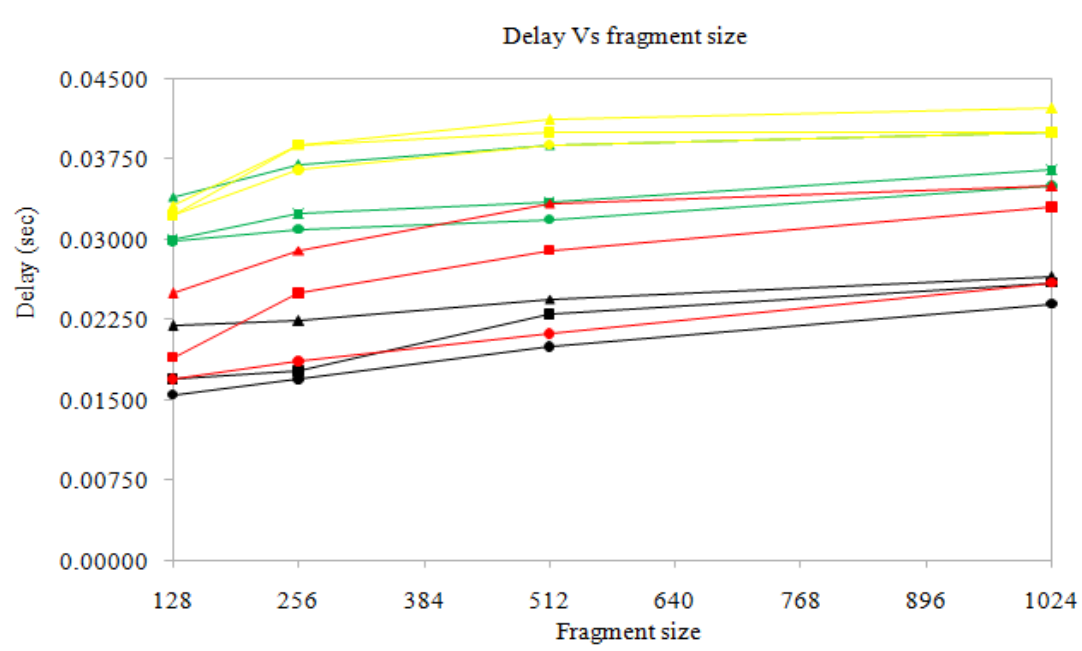

—-TCP_BT_BER $=10 \mathrm{e}-4$

$\longrightarrow$ TCP_BT_BER $=10 \mathrm{e}-5$

$\because$ TCP_BT_BER=10e-6

$\longrightarrow$ TCP_BTDF_BER $=10 \mathrm{e}-4$

$\rightarrow-T C P \_B T D F \_B E R=10 \mathrm{e}-5$

$\longrightarrow$ TCP_BTDF_BER $=10 \mathrm{e}-6$ TCP_DCF_BER $=10 \mathrm{e}-4$ TCP_DCF_BER=10e-5 TCP_DCF_BER $=10 \mathrm{e}-6$

$\longrightarrow$ TCP_AFR_BER $=10 \mathrm{e}-4$

$\longrightarrow$ TCP_AFR_BER $=10 \mathrm{e}-5$

$\longrightarrow$ TCP_AFR_BER $=10 \mathrm{e}-6$

Fig. 19. Delay compared with subframes
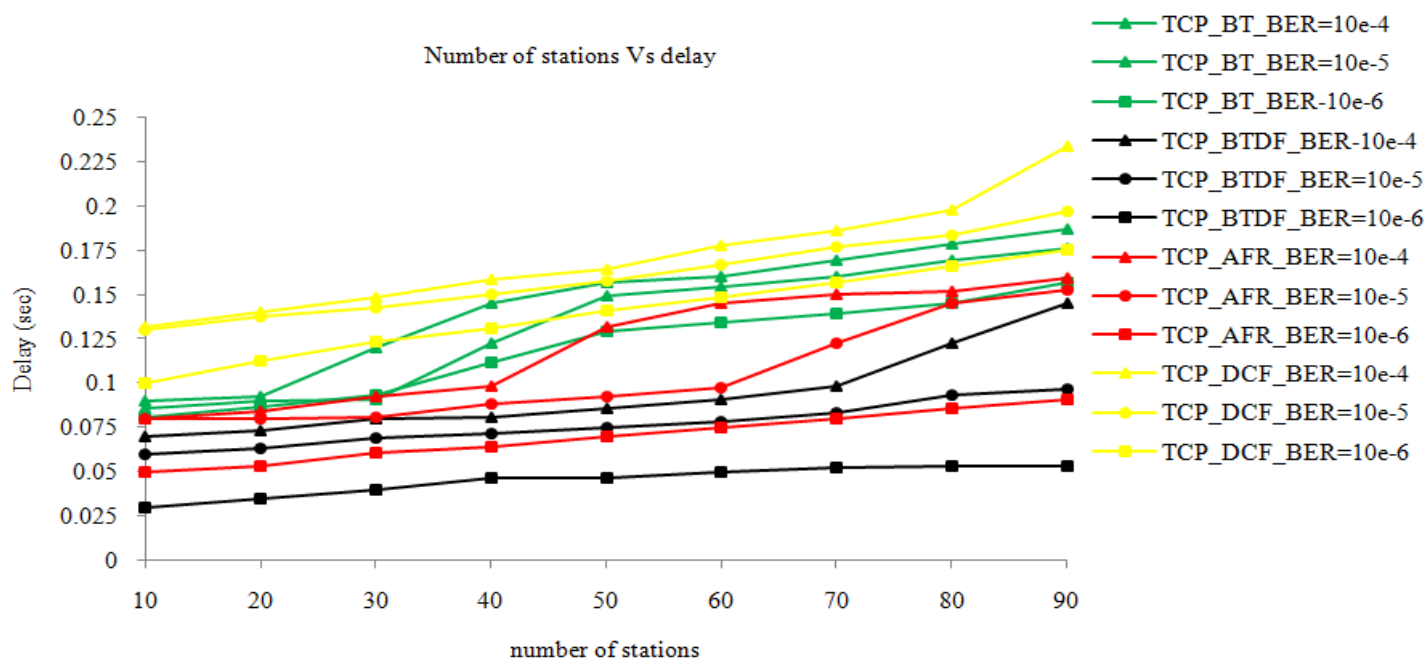

Fig. 20. Delay compared with number of stations

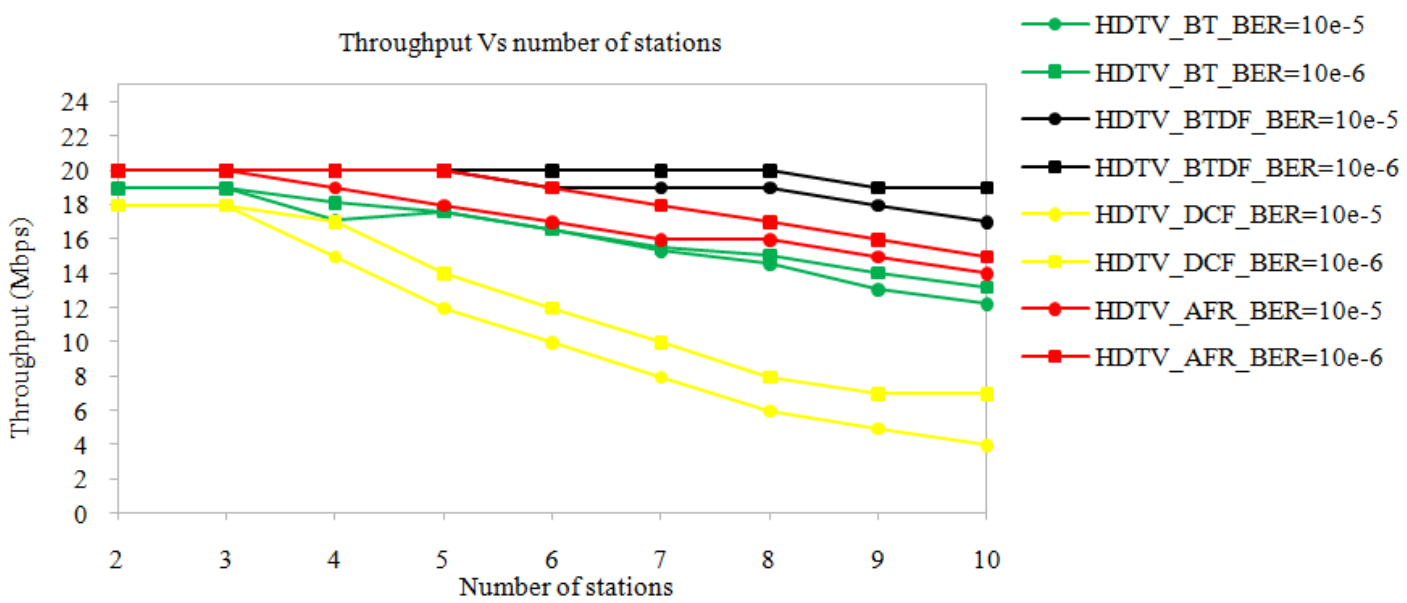

Fig. 21. Throughput compared with number of stations 


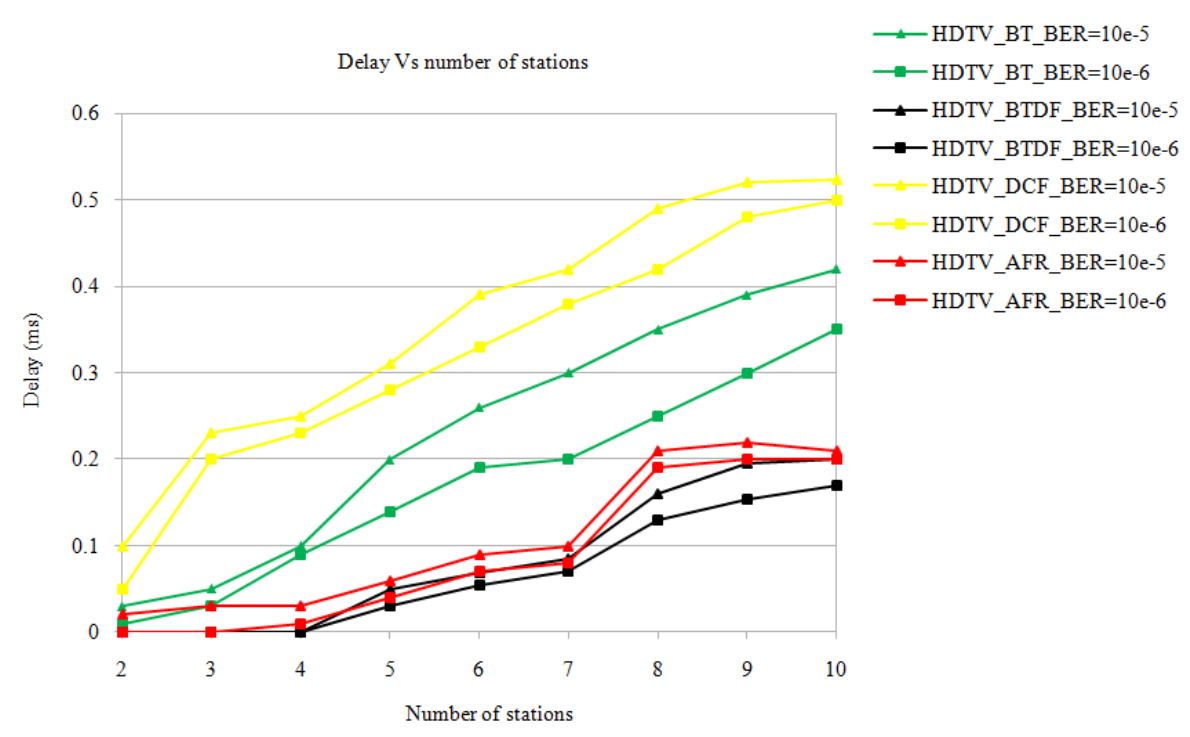

Fig. 22. Delay compared with number of stations

\section{Conclusion}

The bidirectional transmission significantly increased the throughput compared with DCF IEEE802.11. In this study we have proposed a new bidirectional transmission namely BTDF, which are companies the aggregation and bidirectional transmission using block ACK together and we added the fragmentation method to increase the MAC efficiency, only subframes with errors were retransmissted during disruption. The simulation analysis evaluated the throughput and efficiency of BTDF's delay in busy networks and in comparison to identical procedures in previous studies.

\section{Acknowledgement}

This research was funded by the Ministry of Higher Education Malaysia and Universiti Teknikal Malaysia Melaka under Long Term Research Grant LRGS/2015/FTMK/TK01/R0002.

\section{Author's Contributions}

All authors equally contributed in this work.

\section{Ethics}

The article is original and contains unpublished. The corresponding author confirms that all the other authors have read the manuscript and approved it for publication.

\section{References}

Abichar, Z. and J.M. Chang, 2013. Group-Based Medium Access Control for IEEE 802.11n Wireless LANs. IEEE Tran. Mobile Comp., 12: 304-317. DOI: 10.1109/TMC.2011.264
Bruno, R., M. Conti and E. Gregori, 2008. Throughput analysis and measurements in IEEE 802.11 WLANs with TCP and UDP traffic flows. IEEE Tran. Mobile Compu., 7: 171-186. DOI: 10.1109/TMC.2007.70718

Committee WGot, 2009. IEEE Standard for Information technology--Telecommunications and information exchange between systems--Local and metropolitan area networks--Specific requirements Part 11: Wireless LAN Medium Access Control (MAC) and Physical Layer (PHY) Specifications Amendment 5: Enhancements for Higher Throughput. IEEE Std.802, pp: 1-565.

Fayyaz, N., Imranuddin, S.U. Anwar and L. Rukh, 2013. steganography based authentication to prevent IP spoofing. Life Sci. J., 10: 673-677.

Ghaleb, A., D. Chieng, A. Ting, K.H. Kwong and K.C., Lim et al., 2012. Throughput analysis of IEEE802.11n using OPNET. Proceedings of the IET International Conference on Wireless Communications and Applications, Oct. 8-10, IET, Kuala Lumpur, pp: 1-7. DOI: 10.1049/cp.2012.2068

Hajlaoui, N., I. Jabri, M. Taieb and M. Benjemaa, 2012. A frame aggregation scheduler for QoS-sensitive applications in IEEE 802.11n WLANs. Proceedings of the International Conference on Communications and Information Technology, Jun. 26-28, IEEE Xplore press, Hammamet, pp: 221-226.

DOI: 10.1109/ICCITechnol.2012.6285796

Ho, J.M. and D.P. Shaver, 2006. Automatic repeat request for centralized channel access. Google Patents.

Hoefel, R.P.F., 2009. On IEEE EDCA 802.11n. Proceedings of the 6th International Conference on Symposium on Wireless Communication Systems, (WCS'09), IEEE Xplore press, USA, pp: 478-482. 
Chang, Y., C. Lee and J.A. Copeland, 2006. Optimal fragmentation for goodput enhancement in IEEE 802.11 WLAN. Proceedings of the IASTED Conference on Communications, Internet and Information Technology, (IIT' 06), St Thomas, Virgin Islands, pp: 265-270.

Kolap, J., S. Krishnan and N. Shaha, 2012. Frame aggregation mechanism for high-throughput 802.11N WLANS. Int. J. Wireless Mobile Netw., 4: 141-153.

Lee, H.J., J.H. Kim and S.H. Cho, 2007a. A delay-based piggyback scheme in IEEE 802.11. Proceedings of the IEEE Wireless Communications and Networking Conference, Mar. 11-15, IEEE Xplore press, Kowloon, pp: 447-451.

DOI: $10.1109 /$ WCNC.2007.87

Lee, H.J., J.H. Kim and S. Cho, 2007b. A novel piggyback selection scheme in IEEE 802.11e HCCA. Proceedings of the IEEE International Conference on Communications, Jun. 24-28, IEEE Xplore press, Glasgow, pp: 4529-4534. DOI: $10.1109 /$ ICC.2007.748

Lee, T.H., Y.W. Kuo, Y.W. Huang and Y.H. Liu, 2010. To piggyback or not to piggyback acknowledgments? Proceedings of the IEEE 71st Vehicular Technology Conference, May 16-19, IEEE Xplore Press, Taipei, pp: 1-5. DOI: 10.1109/VETECS.2010.5493905

Li, T, Q. Ni, D. Malone, D. Leith and Y. Xiao et al., 2009. Aggregation with fragment retransmission for very high-speed WLANs. IEEE/ACM Tran. Netw., 17: 591-604. DOI: 10.1109/TNET.2009.2014654

Li, T., Q. Ni, D. Malone, D. Leith and Y. Xiao et al., 2006. A new MAC scheme for very high-speed WLANs. Proceedings of the 2006 International Symposium on World of Wireless, Mobile and Multimedia Networks, (MMN' 06), IEEE Computer Society, Washington, pp: 171-180.

DOI: 10.1109/WOWMOM.2006.13

Lim, W.S. and Y.J. Suh, 2010. Achieving per-station fairness in IEEE 802.11 wireless LANs. Proceedings of the IEEE International Symposium on a World of Wireless Mobile and Multimedia Networks, Jun. 1417, IEEE Xplore Press, Canada, pp: 1-9. DOI: 10.1109/WOWMOM.2010.5534908

Lin, Y. and V.W. Wong, 2006. WSN01-1: frame aggregation and optimal frame size adaptation for IEEE 802.11n WLANs. Proceedings of the IEEE Global Telecommunications Conference, Nov. 27Dec. 1, IEEE Xplore Press, San Francisco, pp: 1-6. DOI: 10.1109/GLOCOM.2006.925

Mangold, S., S. Choi, G.R. Hiertz, O. Klein and B. Walkel, 2003. Analysis of IEEE 802.11e for QoS support in wireless LANs. Wireless Commun., 10: 40-50. DOI: 10.1109/MWC.2003.1265851
Milad, A.A., Z.A., Noh, A.S. Shibghatullah and M.A. Algaet, 2013a. Reverse direction transmission in wireless networks: Review. Middle-East J. Scientific Res., 18: 767-778.

Milad, A.A., Z.A.B.M. Noh, A.S. Shibghatullah, S. Sahib and R. Ahmad et al., 2013b. Transmission control protocol performance comparison using piggyback scheme in WLANS. J. Comput. Sci., 9: 967-971. DOI: $10.3844 /$ jcssp. 2013.967.971

Milad, A.A., M.N.Z.A. Bin, A.S. Shibghatullah and M.A., Algaet, 2013c. Design a novel reverse direction transmission using piggyback and piggyback with block ACK to improving the performance of MAC layer based on very high speed wireless LANs. Proceedings of the IEEE Conference on Information and Communication Technologies, Apr. 11-12, IEEE Xplore Press, JeJu Island, pp: 263-266. DOI: $10.1109 /$ CICT.2013.6558102

Milad, A.A., Z.A.B.M. Noh, A.S. Shibghatullah, M.A. Algaet and A. Mustapha, 2014. Reverse direction transmission using single data frame and multi data frames to improve the performance of MAC layer based on IEEE $802.11 \mathrm{n}$. Sci. Int. Lahore, 26: 1861-1864.

Nandhini, R. and N. Devarajan, 2013. Channel quality based cross-layer scheduling algorithm in Wimax networks. Life Sci. J., 10: 2498-2506.

Ozdemir, M., D. Gu, A.B. McDonald and J. Zhang, 2006. Enhancing MAC performance with a reverse direction protocol for high-capacity wireless LANs. Proceedings of the IEEE 64th Vehicular Technology Conference, Sept. 25-28, IEEE Xplore Press, Montreal, pp: 1-5. DOI: 10.1109/VTCF.2006.461

Park, J.S., T.O. Kim, K.J. Kim and B.D. Choi, 2009. Performance analysis of IEEE 802.15.4 Non-beacon mode where downlink data packets are transmitted by piggyback method. Proceedings of the IEEE International Conference on Communications Workshops, Jun. 14-18, IEEE Xplore Press, Dresden, pp: 1-6.

DOI: $10.1109 / \mathrm{ICCW} .2009 .5208114$

Perahia, E. and R. Stacey, 2008. Next Generation Wireless LANs. 1st Edn., Cambridge University Press, Cambridge,

Perahia, E., 2008. IEEE 802.11n development: History, process and technology. Commun. Magazine, 46: 48-55. DOI: 10.1109/MCOM.2008.4557042

Rasheed, M.M., O. Ghazali and R. Budiarto, 2012. Fast Detection of Stealth and Slow Scanning Worms in Transmission Control Protocol. J. Applied Sci., 12: 2156-2163. DOI: 10.3923/jas.2012.2156.2163

Saif, A. and M. Othman, 2013. SRA-MSDU: Enhanced A-MSDU frame aggregation with selective retransmission in $802.11 \mathrm{n}$ wireless networks. J. Netw. Comp. Appli., 36: 1219-1229.

DOI: $10.1016 /$ j.jnca.2013.02.023 
Saif, A., M. Othman, S. Subramaniam and N.A. Abdul Hamid, 2011. Frame aggregation in wireless Networks: Techniques and Issues. IETE Technical Rev., 28: 336-350. 10.4103/0256-4602.83554

Saif, A., M. Othman, S. Subramaniam and N.A.W.A. Hamid, 2012. An enhanced a-msdu frame aggregation scheme for 802.11 n wireless networks. Wireless Personal Commun., 66: 683-706.

DOI: $10.1007 / \mathrm{s} 11277-011-0358-8$

Skordoulis, D., Q. Ni, H.H. Chen, A.P. Stephens and C. Liu, 2008. IEEE 802.11n MAC frame aggregation mechanisms for next-generation high-throughput WLANs. IEEE Wireless Commun, 15: 40-47. DOI: 10.1109/MWC.2008.4454703

Soni, V. and R. Mendiratta, 2008. Next-generation WLAN architecture for high performance networks. Proceedings of the Conference on Wireless, Mobile and Multimedia Networks, Jan. 11-12, Institution of Engineering and Technology, India, pp: 125-129. DOI: $10.1049 / \mathrm{cp}: 20080161$

Tam, A.T. and C.L. Wang, 2003. Contention-aware communicate on schedule for high-speed communication. Cluster Comput., 6: 339-353.

DOI: $10.1023 / \mathrm{A}: 1025765910100$
Wang, C.Y. and H.Y. Wei, 2009. IEEE 802.11n MAC enhancement and performance evaluation. Mobile Netw. Applic., 14: 760-771.

DOI: $10.1007 / \mathrm{s} 11036-008-0129-2$

Xiao, Y. and J. Rosdahl, 2002. Throughput and delay limits of IEEE 802.11. Commun. Lett., 6: 355-357. DOI: 10.1109/LCOMM.2002.802035

Xiao, Y. and J. Rosdahl, 2003. Performance analysis and enhancement for the current and future IEEE 802.11 MAC protocols. ACM SIGMOBILE Mobile Comp. Communi. Rev., 7: 6-19. DOI: $10.1145 / 950391.950396$

Xiao, Y., 2005. IEEE 802.11n: Enhancements for higher throughput in wireless LANs. Wireless Commun., 12: 82-91. DOI: 10.1109/MWC.2005.1561948

Zhai, H., Y. Kwon and Y. Fang, 2004. Performance analysis of IEEE 802.11 MAC protocols in wireless LANs. Wireless Commun. Mobile Comp., 4: 917-931. DOI: $10.1002 / \mathrm{wcm} .263$ 